Recepción: 05/02/2019

Aceptación: 11/03/2019

Publicación: 05/04/2019

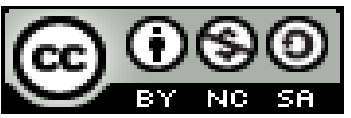

Ciencias técnicas y aplicadas

Artículo de investigación

\title{
Valoración cualitativa de una plantación de Eucalyptus globulus Labill en el sector de Licto, Riobamba, Ecuador
}

\section{Qualitative evaluation of a plantation of Eucalyptus globulus Labill in the sector of Licto, Riobamba, Ecuador}

\section{Evaluación cualitativa de una plantación de Eucalyptus globulus Labill en el sector de Licto, Riobamba, Ecuador}

Miguel Ángel Guallpa-Calva I
miguel.guallpa@espoch.edu.ec

Norma Ximena Lara-Vasconez ${ }^{\text {II }}$

nlara@espoch.edu.ec

Víctor Manuel Espinoza ${ }^{\text {III }}$

victor.espinoza@espoch.edu.ec

\author{
Edmundo Danilo Guilcapi-Pacheco IV \\ eguilcapi@espoch.edu.ec
}

Osvaldo Alberto Fosado-Téllez V

osvaldo.fosado@gmail.com

Correspondencia: miguel.guallpa@espoch.edu.ec

I Magíster en Formulación, Evaluación y Gerencia de Proyectos para el Desarrollo, Magister en Manejo Forestal Sostenible, Ingeniero Forestal, Tecnólogo Agroforestal, Docente Escuela Politécnica de Chimborazo ESPOCH, Riobamba, Ecuador.

II Diplomado Superior en Gestión del Aprendizaje Universitario, Magister en Ciencias, Ingeniera Forestal, Docente Escuela Politécnica de Chimborazo ESPOCH, Riobamba, Ecuador.

III Magíster en Formulación, Evaluación y Gerencia de Proyectos Para el Desarrollo, Ingeniero Forestal, Docente Investigador de la Escuela Politécnica de Chimborazo ESPOCH, Riobamba, Ecuador.

IV Magíster en Producción Animal, Diploma Superior en Investigación y Proyectos, Ingeniero Agrónomo, Docente Escuela Politécnica de Chimborazo ESPOCH, Riobamba, Ecuador.

V Doctor en Ciencias Forestales, Docente Investigador de la Universidad Técnica de Manabí, Portoviejo, Ecuador. 


\title{
Resumen
}

El estudio evalúa de forma cualitativa a los árboles que forman parte de la plantación de Eucalyptus globulus Labill en la Estación Experimental Tunshi-ESPOCH, con el propósito de disponer, indicadores que orienten la aplicación de técnicas silvícolas; es la razón que motivó evaluar la calidad de los árboles en pie por rodal. Mediante el muestreo sistemático no alineado con una intensidad del 5-6\% $\mathrm{ha}^{-1}$, con base en la instalación de parcelas circulares de $500 \mathrm{~m}^{2}$. Dentro de cada parcela se registró ocho criterios de calidad de los árboles. A cada criterio se le asignó el valor de 0 que significa ausencia y 1 para representar la presencia de la característica durante la fase de muestreo y al elaborar la base de datos. La aplicación de tablas de contingencia para el análisis de los residuos corregidos, al comparar los valores absolutos de ausencia o presencia, siendo significativo al $5 \%$, cuando el valor absoluto es $>1,96$. Los resultados de esta investigación muestran cinco asociaciones significativas con la prueba de Chi-cuadrado de Pearson y dos con el Test de Fisher. Finalmente, con la formación de conglomerados jerárquicos, se determinó, un primer grupo formado por los rodales 1,3 y 4; por su parte el rodal 7 presentó características similares al primer grupo, un segundo grupo concentra a los rodales 5 y 6 . En cambio el rodal 2 mostró una condición de rodal atípico. La agrupación de rodales sustenta la toma de decisiones para el manejo sostenible de la plantación en estudio.

Palabras clave: Calidad del árbol; rodal; plantación forestal; Eucalyptus globulus.

\begin{abstract}
The study qualitatively evaluates the trees that are part of the plantation of Eucalyptus globulus Labill in the Tunshi-ESPOCH Experimental Station, with the purpose of having indicators that guide the application of silvicultural techniques; This is the reason that motivated the evaluation of the quality of standing trees per stand. Through systematic sampling not aligned with an intensity of 5-6\% ha-1, based on the installation of circular plots of $500 \mathrm{~m} 2$. Within each plot, eight tree quality criteria were recorded. Each criterion was assigned the value of 0 which means absence and 1 to represent the presence of the characteristic during the sampling phase and when preparing the database. The application of contingency tables for the analysis of the corrected residuals, when comparing the absolute values of absence or presence, being significant at $5 \%$, when the absolute value is $>1.96$. The results of this investigation show five significant
\end{abstract}


associations with the Pearson Chi-square test and two with the Fisher test. Finally, with the formation of hierarchical conglomerates, a first group formed by stands 1, 3 and 4 was determined; On the other hand, stand 7 presented similar characteristics to the first group, a second group concentrated on stands 5 and 6. In contrast, stand 2 showed an atypical stand condition. The grouping of stands supports the decision making for the sustainable management of the plantation under study.

Keywords: Quality of the tree; stand; forest plantation; Eucalyptus globulus.

\section{Resumo}

O estudo avaliou árvores qualitativamente como parte de plantar Eucalyptus globulus Labill na Estação Experimental Tunshi-ESPOCH, a fim de proporcionar indicadores para orientar a aplicação de técnicas de silvicultura; Esse é o motivo que motivou a avaliação da qualidade das árvores em pé por estande. Através de amostragem sistemática não alinhada com uma intensidade de 5-6\% ha-1, baseada na instalação de parcelas circulares de $500 \mathrm{~m} 2$. Dentro de cada parcela, oito critérios de qualidade das árvores foram registrados. A cada critério foi atribuído o valor de 0, que significa ausência e 1 para representar a presença da característica durante a fase de amostragem e ao preparar o banco de dados. A aplicação de tabelas de contingência para a análise dos resíduos corrigidos, quando se comparam os valores absolutos de ausência ou presença, é significativa a 5\%, quando o valor absoluto é> 1,96. Os resultados desta investigação mostram cinco associações significativas com o teste Qui-quadrado de Pearson e dois com o teste de Fisher. Finalmente, com a formação de conglomerados hierárquicos, determinou-se um primeiro grupo formado pelos estandes 1,3 e 4; Por seu turno, o estande 7 apresentou características semelhantes ao primeiro grupo, um segundo grupo concentra os estandes 5 e 6 . Em contrapartida, o estande 2 apresentou uma condição de estagnação atípica. $\mathrm{O}$ agrupamento de estandes apóia a tomada de decisão para o manejo sustentável da plantação em estudo.

Palavras-chave: Qualidade da árvore; ficar de pé plantação florestal; Eucalyptus globulus. 


\section{Introducción}

El eucalipto es una especie de alta capacidad productiva que prospera en terrenos forestales degradados o inutilizados, se desarrolla en condiciones edafoclimáticas diversas (ENCE, 2009). A nivel de Ecuador, es priorizada en la región serrana para la forestación con fines comerciales debido a su adaptabilidad, rápido crecimiento y rentabilidad (MAGAP, 2015).

La evaluación de la calidad de las plantaciones, aplica una metodología que asigna una calidad en función de distintas variables cualitativas que pueden determinar el valor de los árboles en pie. Con base en parámetros que permitan conocer el estado actual de la calidad de los árboles y poder comparar diferentes rodales. Dado que la calidad de las plantaciones depende de la combinación entre la selección del sitio de plantación de acuerdo con la especie utilizada, del uso de material genético de calidad y del manejo silvicultural aplicado a la plantación (Murillo y Badilla, 2010). La influencia de los tratamientos silvícolas sobre la calidad de los árboles, es un elemento primordial para analizar a partir del levantamiento de información de los árboles con el propósito de plantear esquemas de manejo silvicultural (Jiménez, 2008).

En la provincia de Chimborazo, la mayoría de evaluaciones de plantaciones forestales están orientadas específicamente al cálculo del volumen de madera en pie, y en ciertos casos la información de calidad es manejada de manera particular por ciertas empresas o propietarios de masas forestales. La plantación de E. globulus es parte de la Estación Experimental Tunshi perteneciente a la Escuela Superior Politécnica de Chimborazo, entre sus principales funciones: por una parte, contribuye a la protección del equilibrio del ambiente y por otra es proveer de espacios para la realización de prácticas e investigaciones académicas. Fue importante calificar los atributos al observar a los árboles de 7 rodales que conforman la masa forestal en estudio.

Partiendo del hecho, que la productividad y calidad de los árboles de eucalipto a nivel de sitio y micro sitio en el sector de Tunshi, en condiciones adversas, y la ausencia de labores silviculturales ejercen influencia sobre los atributos de los árboles en pie, motivó valorar su calidad, para disponer de su tipificación de forma cualitativa como soporte adicional para la toma de decisiones de manejo técnico.

Con base en el muestreo del bosque plantado, se recopiló la información considerando una variable clasificatoria de calidad de los árboles, misma que agrupa a criterios seleccionados 
(Nájera \& Hernández, 2008). El objetivo del presente estudio fue evaluar las características de los árboles en pie de cada rodal. A fin de disponer de indicadores que orienten la aplicación de técnicas silvícolas, para mejorar su productividad, o la decisión de uso futuro de las áreas con bosque plantado (Alvarado, 2013).

\section{Materiales y Métodos}

La investigación se realizó en la plantación de E. globulus Labill de 38 años de edad, ubicada en la Estación Experimental Tunshi de la Escuela Superior Politécnica de Chimborazo, Tunshi Grande, parroquia Licto, al sur oeste del cantón Riobamba, provincia de Chimborazo-Ecuador, a $12 \mathrm{~km}$ de la ciudad capital. Geográficamente la masa forestal se inserta dentro de los puntos de referencia: UTM Zona 17S Datum WGS $84 \mathrm{X}_{1}=763609 ; \mathrm{Y}_{1}=9806880, \mathrm{X}_{2}=764973 ; \mathrm{Y}_{2}=$ 9806791, $X_{3}=764973 ; Y_{3}=9805248$ y $X_{4}=763606 ; Y_{4}=9805251$. Se sitúa a una altitud que va desde 2755 - $2929 \mathrm{msnm}$. La precipitación anual es 636,4 mm, siendo la temperatura media $14,5^{\circ} \mathrm{C}$ y una humedad relativa media de $85 \%$. Datos de la Estación Guaslan; serie 1990-2014; MAGAP. La plantación tiene una superficie neta de 37,62 ha, un área sin vegetación de 1,38 has dando un total de 39,00 ha y se categoriza dentro de la formación: estepa Espinoza-Montano Bajo (Sierra et al., 2012).

La Tabla 1 muestra las características fisiográficas y del suelo del área de plantación reportadas en el estudio desarrollado por (Guallpa, Rosero, Samaniego, \& Cevallos, 2016).

Tabla 1. Principales características fisiográficas y del suelo en el área de estudio

\begin{tabular}{|c|c|c|c|c|}
\hline Rodal & $\begin{array}{l}\text { Altitud } \\
\text { (msnm) }\end{array}$ & $\begin{array}{l}\text { Pendiente } \\
(\%)\end{array}$ & $\begin{array}{c}\text { Nivel de pedregosidad } \\
\text { interna }(\%)\end{array}$ & $\begin{array}{c}\text { Nivel de drenaje interno } \\
\left(\mathrm{cm} \cdot \mathrm{h}^{-1}\right)\end{array}$ \\
\hline 1 & 2787 & 20 & No pedregoso & Drenaje moderado \\
\hline 2 & 2861 & 55 & No pedregoso & Drenaje moderadamente lento \\
\hline 3 & 2929 & 55 & No pedregoso & Drenaje moderadamente lento \\
\hline 4 & 2894 & 58 & Moderadamente pedregoso & Drenaje moderadamente lento \\
\hline 5 & 2793 & 57 & No pedregoso & Drenaje moderadamente lento \\
\hline 6 & 2774 & 50 & No pedregoso & Drenaje moderado \\
\hline 7 & 2755 & 18 & Moderadamente pedregoso & Drenaje moderado \\
\hline
\end{tabular}

La caracterización de las variables dasométricas de la plantación de E. globulus Labill realizada 
en el año 2014 reportó en estado fustal con un DAP >a 10cm, 441.13 árboles ha ${ }^{-1}$; un DAP promedio de $23.17 \mathrm{~cm}$, y una altura total de $21.23 \mathrm{~m}$. La media aritmética del área basal de los siete rodales fue d $0,54 \mathrm{~m}^{2}$ árbol ${ }^{-1}$, con 441.13 árboles, se estima $22.21 \mathrm{~m}^{2} \mathrm{ha}^{-1}$ de área basal y una densidad en cobertura del $96.47 \%$ de área basal con un volumen total de $472.09 \mathrm{~m}^{3} \mathrm{ha}^{-1}$. El estudio dasométrico con las variables; área basal $\mathrm{ha}^{-1}$, la densidad de cobertura en $\%$ de los 7 rodales y el volumen total promedio árbol ${ }^{-1}$, determino la asociación entre rodales, el primer grupo conformado por los rodales 1, 2, 3 y 4 y el segundo por los rodales 5, 6 y 7 (Guallpa et al., op.cit, p., 8).

\section{Muestreo}

El muestreo de calidad de la plantación de E. globulus tuvo lugar en el último semestre del 2017. Para evaluar los rodales de la plantación se aplicó un muestreo sistemático no alineado con una intensidad de15-6 \% ha ${ }^{-1}$, mediante la instalación de parcelas circulares con un radio de $12.62 \mathrm{~m}$, distanciadas entre ellas de 75 y 100 m, en base a la topografía y exposición de cada rodal (FAO, 2011).

\section{Evaluación de la plantación}

Se fundamentó en la asignación de calidad en función de distintas variables cualitativas seleccionadas para determinar las características de los árboles en pie al considerar ciertas variables de la metodología desarrollada por Murillo y Badilla, (2010). Así como en la propuesta de procedimientos para el seguimiento de parcelas permanentes, mediante el registro de criterios considerados en las evaluaciones de campo de los árboles valorados dentro de cada parcela (Salazar, 2008; ETIFN, 2014).

\section{Variable clasificatoria}

El estudio parte de la determinación de los criterios más influyentes en la calidad árboles en función del rodal en el que se encuentran. La valoración analítica se agrupa en una característica clasificatoria (Tabla 2). Cada árbol es considerado como una "unidad de registro" a la que se le asigna una clase de calidad. La evaluación se realizó de forma separada de las diferentes características y la subsecuente reagrupación de las valoraciones. Tales criterios, se resume en la Tabla 2, de acuerdo a lo sugerido por (Samaniego, 2013; González, 2017). 
Tabla 2. Variable clasificatoria de calidad de los árboles en pie

\begin{tabular}{|l|l|l|}
\hline \multicolumn{1}{|c|}{ Código } & \multicolumn{1}{|c|}{ Variable } & \multicolumn{1}{c|}{ Descripción } \\
\hline Ps & Poco sinuoso & El fuste presenta curvaturas o alabeos leves \\
\hline $\mathrm{Ms}$ & Muy sinuoso & El fuste presenta curvaturas o alabeos severos \\
\hline $\mathrm{Tb}$ & Torcedura basal & $\begin{array}{l}\text { Curvatura basal brusca, lo que disminuye la longitud } \\
\text { útil del fuste }\end{array}$ \\
\hline $\mathrm{Bf}$ & Bifurcado & $\begin{array}{l}\text { Hábito de crecer en forma de horquetas que origina la } \\
\text { formación de } 2 \text { o más fustes }\end{array}$ \\
\hline $\mathrm{Li}$ & $\begin{array}{l}\text { Ligeramente } \\
\text { inclinado }\end{array}$ & $\begin{array}{l}\text { Si forma un ángulo menor a } 90^{\circ} \text { o igual a } 80^{\circ} \text { con } \\
\text { relación al suelo }\end{array}$ \\
\hline $\mathrm{I}$ & Inclinado & $\begin{array}{l}\text { Un árbol se considera inclinado cuando su eje } \\
\text { longitudinal forma un ángulo menor de } 80^{\circ} \text { o igual a } \\
60^{\circ} \text { con la horizontal }\end{array}$ \\
\hline $\mathrm{Sin}$ copa & Ausencia de copa & El árbol no presenta una copa \\
\hline Fr & Fuste recto & El fuste forma un ángulo de $90^{\circ}$ con relación al suelo \\
\hline
\end{tabular}

Todas estas variables son binarias, donde se asignó el valor 0 que significa ausencia y 1 presencia de la característica evaluada durante la fase de muestreo.

\section{Análisis estadístico}

Se elaboró una base de datos para el análisis de las variables cualitativas. Utilizando el programa estadístico SPSS Statistics 22 (Nel, 2014). Con base en el análisis individual de cada variable con el objetivo de determinar si existe dependencia o no de cada una de las características con respecto al rodal. Se utilizó las tablas de contingencia que incorpora los residuos corregidos y la prueba de Chi-cuadrado de Pearson aplicada a cada análisis de contingencia para determinar la mayor o menor asociación de cada atributo por rodal, estos métodos permiten, determinar el sentido y quién provoca esa dependencia (De la Fuente-Fernández, 2011).

Los residuos son las diferencias entre la frecuencia observada y la frecuencia esperada en cada casilla: $e_{i j}=N_{i j}-E_{i j}$. El análisis de los residuos en las tablas de contingencia fue sugerido por Haberman y consiste en estudiar su patrón; pero dado que generalmente no son sencillos de observar se recurre a la comparación de los residuos ajustados a una distribución teórica conocida: 


\section{Residuos ajustados}

$e_{i j}=\frac{N_{i j}-E_{i j}}{\sqrt{E_{i j}}}$

$N_{i j}:$ Frecuencia observada.

$E_{i j}$ : Frecuencia teórica.

Residuos tipificados

$$
\begin{aligned}
& d_{i j}=\frac{\left(N_{i j}-E_{i j}\right) / \sqrt{E_{i j}}}{\sqrt{V_{i j}}}=\frac{e_{i j}}{\sqrt{V_{i j}}} \\
& V_{i j}=V\left(e_{i j}\right)=\left(1-\frac{n_{i j}}{N}\right)\left(1-\frac{n_{j j}}{N}\right)
\end{aligned}
$$

Cuando los dos factores objeto de estudio son independientes, cada residuo tipificado $\mathrm{d}_{\mathrm{ij}}$ se distribuye asintóticamente como una $\mathrm{N}(0,1)$

1. Para un nivel de significancia del 5\% se comparan los valores absolutos de la tabla de entrada de los residuos corregidos. Es significativo siempre que su valor absoluto supere 1,96 .

2. En los valores que cumplen con la condición se analiza el signo, lo que permite conocer el sentido de dicha relación (García, 2016).

Para la interpretación del Test Chi-cuadrado de Pearson, los datos son compatibles con la hipótesis de independencia, si la probabilidad asociada al estadístico $\mathrm{x}^{2}$ es alta cuando el p-valor es $>0,05, \quad$ y $\quad$ si $\quad$ es $<0.05$, conduce a rechazar la hipótesis nula, concluyendo que existe dependencia o relación entre las variables analizadas. También se toma en cuenta, si existen frecuencias esperadas menores que 5, éstas no deben superar el 20 por ciento del total de frecuencias esperadas. Caso contrario se usó el Test de Fisher (López y Fachelli, 2015). Finalmente, se realizó la reagrupación de las características de calidad de los árboles en pie al conformar grupos de rodales con características similares (De la Fuente-Fernández, 2011a). 


\section{Resultados y Discusión}

\section{Calidad de los árboles. Análisis de contingencia de la relación poco sinuosa de los árboles en pie por rodal}

El análisis de los residuos corregidos para la variable poco sinuosos de los árboles en pie por rodal, indica que existe dependencia provocado por una tendencia al incremento en el rodal 4 en su valor de ausencia $(4,5)$ y decremento en su valor de presencia $(-4,5)$ en referencia a la característica evaluada. En el caso del rodal 5 ocurre lo contrario, hay un decremento de su valor de ausencia $(-3,6)$ e incremento en su valor de presencia $(3,6)$ reportado. En el resto de los rodales no se observa dependencia significativa según el análisis de los residuos a un nivel de confianza de 95\%, tal como se observa en la Tabla 3.

Tabla 3. Análisis de contingencia de la variable poco sinuosa de los árboles en pie por rodal

\begin{tabular}{|c|c|c|c|c|c|}
\hline & \multicolumn{2}{|c|}{ Poco sinuosos } & \multirow[b]{2}{*}{ Total } \\
\hline & & & Ausencia (0) & $\begin{array}{c}\text { Presencia } \\
\text { (1) }\end{array}$ & \\
\hline \multirow{21}{*}{$\begin{array}{l}\text { Roda } \\
1\end{array}$} & \multirow{3}{*}{1} & Recuento & 299 & 103 & 402 \\
\hline & & Frecuencia esperada & 304,2 & 97,8 & 402,0 \\
\hline & & Residuos corregidos &,- 8 & 8 & \\
\hline & \multirow{3}{*}{2} & Recuento & 101 & 27 & 128 \\
\hline & & Frecuencia esperada & 96,9 & 31,1 & 128,0 \\
\hline & & Residuos corregidos & ,9 &,- 9 & \\
\hline & \multirow{3}{*}{3[} & Recuento & 26 & 6 & 32 \\
\hline & & Frecuencia esperada & 24,2 & 7,8 & 32,0 \\
\hline & & Residuos corregidos &, 7 &,- 7 & \\
\hline & \multirow{3}{*}{4} & Recuento & 200 & 30 & 230 \\
\hline & & Frecuencia esperada & 174,0 & 56,0 & 230,0 \\
\hline & & Residuos corregidos & 4,5 & $-4,5$ & \\
\hline & \multirow{3}{*}{5} & Recuento & 126 & 66 & 192 \\
\hline & & Frecuencia esperada & 145,3 & 46,7 & 192,0 \\
\hline & & Residuos corregidos & $-3,6$ & 3,6 & \\
\hline & \multirow{3}{*}{6} & Recuento & 56 & 21 & 77 \\
\hline & & Frecuencia esperada & 58,3 & 18,7 & 77,0 \\
\hline & & Residuos corregidos &,- 6 & ,6 & \\
\hline & \multirow{3}{*}{7} & Recuento & 35 & 18 & 53 \\
\hline & & Frecuencia esperada & 40,1 & 12,9 & 53,0 \\
\hline & & Residuos corregidos & $-1,7$ & 1,7 & \\
\hline \multirow{2}{*}{ Total } & & Recuento & 843 & 271 & 1114 \\
\hline & & Frecuencia esperada & 843,0 & 271,0 & 1114,0 \\
\hline
\end{tabular}

\section{4}

Pol. Con. (Edición núm. 32) Vol. 4, No 4, abril 2019, pp. 126-152, ISSN: 2550 - 682X 
A través del análisis del Test Chi-cuadrado de Pearson para la Tabla 3, se evidencia una relación de dependencia entre la variable poco sinuosa de los árboles en pie por rodal ( $p$-valor $<\alpha=0.05$; Tabla 4).

Tabla 4. Pruebas de Chi-cuadrado de la tabla de contingencia de la variable poco sinuosa de los

\begin{tabular}{|c|c|c|c|c|c|c|}
\hline \multicolumn{7}{|c|}{ árboles en pie por rodal } \\
\hline & Valor & $\mathrm{gl}$ & $\begin{array}{c}\text { Sig. } \\
\text { asintótica } \\
\text { (bilateral) }\end{array}$ & $\begin{array}{c}\text { Sig. exacta } \\
\text { (bilateral) }\end{array}$ & $\begin{array}{l}\text { Sig. exacta } \\
\text { (unilateral) }\end{array}$ & $\begin{array}{l}\text { Probabilidad } \\
\text { en el punto }\end{array}$ \\
\hline Chi-cuadrado de Pearson & $31,107^{\mathrm{a}}$ & 6 &, 000 &.$^{b}$ & & \\
\hline Razón de verosimilitudes & 32,451 & 6 & 000 &. $\mathrm{~b}$ & & \\
\hline $\begin{array}{l}\text { Estadístico exacto de } \\
\text { Fisher }\end{array}$ & & & &.$^{\mathrm{b}}$ & & \\
\hline \begin{tabular}{|l|} 
Asociación lineal por \\
lineal
\end{tabular} & $1,437^{\mathrm{c}}$ & 1 & ,231 & 232 &, 119 & ,007 \\
\hline $\mathrm{N}$ de casos válidos & 1114 & & & & & \\
\hline
\end{tabular}

a. 0 casillas $(0,0 \%)$ tienen una frecuencia esperada inferior a 5 . La frecuencia mínima esperada es 7,78.

b. No se puede efectuar el cálculo porque no hay suficiente memoria.

c. El estadístico tipificado es 1,199 .

\section{Análisis de contingencia de la relación muy sinuosa de los árboles en pie por rodal}

El análisis de los residuos corregidos para la variable muy sinuosa de los árboles en pie por rodal, muestra que existe dependencia provocada por una tendencia al incremento en los rodales 1, 2 y 7 en sus valores de ausencia $(4,3 ; 3,0$ y 2,1$)$ y decremento en sus valores de presencia $(-4,3 ;-3,0$ y $2,1)$ en base a la característica evaluada respectivamente. En el caso de los rodales 5 y 6 ocurre lo contrario, hay un decremento en sus valores de ausencia $(-7,2$ y $-3,9)$ e incremento en sus valores de presencia (7,2 y 3,9; Tabla 5). 
Tabla 5. Análisis de contingencia de la variable muy sinuosa de los árboles en pie por rodal

\begin{tabular}{|c|c|c|c|c|c|}
\hline & \multicolumn{2}{|c|}{ Muy sinuoso } & \multirow{2}{*}{ Total } \\
\hline & & & $\begin{array}{c}\text { Ausencia } \\
(0)\end{array}$ & \multirow{2}{*}{\begin{tabular}{|r|} 
Presencia (1) \\
11 \\
\end{tabular}} & \\
\hline \multirow{21}{*}{ Rodal } & \multirow{3}{*}{1} & Recuento & 391 & & 402 \\
\hline & & Frecuencia esperada & 373,5 & 28,5 & 402,0 \\
\hline & & Residuos corregidos & 4,3 & $-4,3$ & \\
\hline & \multirow{3}{*}{2} & Recuento & 127 & 1 & 128 \\
\hline & & Frecuencia esperada & 118,9 & 9,1 & 128,0 \\
\hline & & Residuos corregidos & $\mathbf{3 , 0}$ & $-3,0$ & \\
\hline & \multirow{3}{*}{3} & Recuento & 32 & 0 & 32 \\
\hline & & Frecuencia esperada & 29,7 & 2,3 & 32,0 \\
\hline & & Residuos corregidos & 1,6 & $-1,6$ & \\
\hline & \multirow{3}{*}{4} & Recuento & 214 & 16 & 230 \\
\hline & & Frecuencia esperada & 213,7 & 16,3 & 230,0 \\
\hline & & Residuos corregidos &, 1 &,- 1 & \\
\hline & \multirow{3}{*}{5} & Recuento & 155 & 37 & 192 \\
\hline & & Frecuencia esperada & 178,4 & 13,6 & 192,0 \\
\hline & & Residuos corregidos & $-7,2$ & 7,2 & \\
\hline & \multirow{3}{*}{6} & Recuento & 63 & 14 & 77 \\
\hline & & Frecuencia esperada & 71,5 & 5,5 & 77,0 \\
\hline & & Residuos corregidos & $-3,9$ & 3,9 & \\
\hline & \multirow{3}{*}{7} & Recuento & 53 & 0 & 53 \\
\hline & & Frecuencia esperada & 49,2 & 3,8 & 53,0 \\
\hline & & Residuos corregidos & 2,1 & $-2,1$ & \\
\hline \multirow{2}{*}{ Total } & & Recuento & 1035 & 79 & 1114 \\
\hline & & Frecuencia esperada & 1035,0 & 79,0 & 1114,0 \\
\hline
\end{tabular}

En el caso del análisis de la relación de la variable muy sinuosa de los árboles en pie por rodal, el número de casillas con valores esperados inferiores a 5 , no sobrepasan el $20 \%$ de las frecuencias esperadas, donde la prueba Chi-cuadrado de Pearson determinó una asociación significativa a un nivel de confianza de $95 \%$ ( $\mathrm{p}$-valor $<\alpha=0.05$; Tabla 6 ). 
Tabla 6. Pruebas de Chi-cuadrado de la tabla de contingencia de la variable muy sinuosa de los árboles en pie por rodal

\begin{tabular}{|c|c|c|c|c|c|c|}
\hline & Valor & $\mathrm{gl}$ & $\begin{array}{c}\text { Sig. } \\
\text { asintótica } \\
\text { (bilateral) }\end{array}$ & $\begin{array}{l}\text { Sig. exacta } \\
\text { (bilateral) }\end{array}$ & $\begin{array}{l}\text { Sig. exacta } \\
\text { (unilateral) }\end{array}$ & $\begin{array}{c}\text { Probabilidad } \\
\text { en el punto }\end{array}$ \\
\hline Chi-cuadrado de Pearson & $83,404^{\mathrm{a}}$ & 6 &, 000 &. & & \\
\hline $\begin{array}{l}\text { Razón de } \\
\text { verosimilitudes }\end{array}$ & 80,428 & 6 &, 000 & $b^{b}$ & & \\
\hline $\begin{array}{l}\text { Estadístico exacto de } \\
\text { Fisher }\end{array}$ & $\mathrm{b}$ & & & $\mathrm{b}$ & & \\
\hline $\begin{array}{l}\text { Asociación lineal por } \\
\text { lineal }\end{array}$ & $35,685^{\mathrm{c}}$ & 1 &, 000 & ,000 & ,000 & ,000 \\
\hline $\mathrm{N}$ de casos válidos & 1114 & & & & & \\
\hline
\end{tabular}

a. 2 casillas (14,3\%) tienen una frecuencia esperada inferior a 5. La frecuencia mínima esperada es 2,27

b. No se puede efectuar el cálculo porque no hay suficiente memoria

c. El estadístico tipificado es 5,974

\section{Análisis de contingencia de la relación torcedura basal de los árboles en pie por rodal}

El análisis de los residuos corregidos para la variable torcedura basal de los árboles en pie por rodal, indica dependencia provocada por una tendencia al incremento en el rodall en su valor de ausencia $(4,2)$ y decremento en su valor de la presencia $(-4,2)$ correspondientes al criterio evaluado. En cambio, en los rodales 5 y 6 ocurre lo contrario, hay un decremento en sus valores de ausencia $(-4,3$ y $-2,1)$ e incremento en sus valores de presencia reportados $(4,3$ y 2,1; Tabla 7$)$. 
Tabla 7. Análisis de contingencia de la variabletorcedura basal de los árboles en pie por rodal

\begin{tabular}{|c|c|c|c|c|c|}
\hline & \multicolumn{2}{|c|}{ Torcedura basal } & \multirow[b]{2}{*}{ Total } \\
\hline & & & $\begin{array}{c}\text { Ausencia } \\
(0)\end{array}$ & \multirow{2}{*}{$\begin{array}{l}\text { Presencia } \\
\text { (1) } \\
\end{array}$} & \\
\hline \multirow{21}{*}{ Rodal } & \multirow{3}{*}{1} & Recuento & 402 & & 402 \\
\hline & & Frecuencia esperada & 391,2 & 10,8 & 402,0 \\
\hline & & Residuos corregidos & 4,2 & $-4,2$ & \\
\hline & \multirow{3}{*}{2} & Recuento & 127 & 1 & 128 \\
\hline & & Frecuencia esperada & 124,6 & 3,4 & 128,0 \\
\hline & & Residuos corregidos & 1,4 & $-1,4$ & \\
\hline & \multirow{3}{*}{3} & Recuento & 32 & 0 & 32 \\
\hline & & Frecuencia esperada & 31,1 & ,9 & 32,0 \\
\hline & & Residuos corregidos & 1,0 & $-1,0$ & \\
\hline & \multirow{3}{*}{4} & Recuento & 220 & 10 & 230 \\
\hline & & Frecuencia esperada & 223,8 & 6,2 & 230,0 \\
\hline & & Residuos corregidos & $-1,7$ & 1,7 & \\
\hline & \multirow{3}{*}{5} & Recuento & 178 & 14 & 192 \\
\hline & & Frecuencia esperada & 186,8 & 5,2 & 192,0 \\
\hline & & Residuos corregidos & $-4,3$ & 4,3 & \\
\hline & \multirow{3}{*}{6} & Recuento & 72 & 5 & 77 \\
\hline & & Frecuencia esperada & 74,9 & 2,1 & 77,0 \\
\hline & & Residuos corregidos & $-2,1$ & 2,1 & \\
\hline & \multirow{3}{*}{7} & Recuento & 53 & 0 & 53 \\
\hline & & Frecuencia esperada & 51,6 & 1,4 & 53,0 \\
\hline & & Residuos corregidos & 1,2 & $-1,2$ & \\
\hline \multirow{2}{*}{ Total } & & Recuento & 1084 & 30 & 1114 \\
\hline & & Frecuencia esperada & 1084,0 & 30,0 & 1114,0 \\
\hline
\end{tabular}

En el caso del análisis de la relación de la variable torcedura basal de los árboles en pie por rodal, el número de casillas con valores esperados inferiores a 5, sobrepasan el 20\% de las frecuencias esperadas (28.6\% de las casillas), por lo que se utilizó el Test de Fisher que sugiere asociación significativa entre la característica torcedura basal y los rodales ( $p$-valor $<\alpha=0.05$; Tabla 8 ). 
Tabla 8. Pruebas de Chi-cuadrado de la tabla de contingencia de la variable Torcedura basal por

\begin{tabular}{|l|r|c|r|r|r|r|}
\hline & Valor & gl & $\begin{array}{c}\text { Sig. } \\
\text { asintótica } \\
\text { (bilateral) }\end{array}$ & $\begin{array}{c}\text { Sig. exacta } \\
\text { (bilateral) }\end{array}$ & $\begin{array}{c}\text { Sig. exacta } \\
\text { (unilateral) }\end{array}$ & $\begin{array}{c}\text { Probabilidad } \\
\text { en el punto }\end{array}$ \\
\hline Chi-cuadrado de Pearson & $37,406^{\mathrm{a}}$ & 6 &, 000 &, 000 & & \\
\hline $\begin{array}{l}\text { Razón de } \\
\text { verosimilitudes }\end{array}$ & 44,809 & 6 &, 000 &, 000 & & \\
\hline $\begin{array}{l}\text { Estadístico exacto de } \\
\text { Fisher }\end{array}$ & 38,039 & & &, 000 & &, 000 \\
\hline $\begin{array}{l}\text { Asociación lineal por } \\
\text { lineal }\end{array}$ & $21,164^{\mathrm{b}}$ & 1 &, 000 &, 000 &, 000 & \\
\hline $\mathrm{N}$ de casos válidos & 1114 & & & & & \\
\hline
\end{tabular}

a. 4 casillas $(28,6 \%)$ tienen una frecuencia esperada inferior a 5 . La frecuencia mínima esperada es ,86.

b. El estadístico tipificado es 4,600.

\section{Análisis de contingencia de la relación bifurcación de los árboles en pie por rodal}

El análisis de los residuos corregidos para la variable bifurcación de los árboles en pie por rodal, indica dependencia provocada por una tendencia al decremento en el rodal 4 en su valor de ausencia $(-4,2)$ e incremento en su valor de presencia $(4,2)$ del criterio evaluado. El resto de los rodales no sugieren dependencia a un nivel de confianza de 95\% (Tabla 9). 
Tabla 9. Análisis de contingencia de la variable bifurcación de los árboles en pie por rodal

\begin{tabular}{|c|c|c|c|c|c|}
\hline & \multicolumn{2}{|c|}{ Bifurcación } & \multirow[b]{2}{*}{ Total } \\
\hline & & & $\begin{array}{c}\text { Ausencia } \\
(0)\end{array}$ & $\begin{array}{c}\text { Presencia } \\
\text { (1) }\end{array}$ & \\
\hline \multirow{21}{*}{$\begin{array}{l}\text { Roda } \\
1\end{array}$} & \multirow{3}{*}{1} & Recuento & 394 & 8 & 402 \\
\hline & & Frecuencia esperada & 390,5 & 11,5 & 402,0 \\
\hline & & Residuos corregidos & 1,3 & $-1,3$ & \\
\hline & \multirow{3}{*}{2} & Recuento & 127 & 1 & 128 \\
\hline & & Frecuencia esperada & 124,3 & 3,7 & 128,0 \\
\hline & & Residuos corregidos & 1,5 & $-1,5$ & \\
\hline & \multirow{3}{*}{3} & Recuento & 31 & 1 & 32 \\
\hline & & Frecuencia esperada & 31,1 & ,9 & 32,0 \\
\hline & & Residuos corregidos &,- 1 & 1 & \\
\hline & \multirow{3}{*}{4} & Recuento & 214 & 16 & 230 \\
\hline & & Frecuencia esperada & 223,4 & 6,6 & 230,0 \\
\hline & & Residuos corregidos & $-4,2$ & 4,2 & \\
\hline & \multirow{3}{*}{5} & Recuento & 188 & 4 & 192 \\
\hline & & Frecuencia esperada & 186,5 & 5,5 & 192,0 \\
\hline & & Residuos corregidos &, 7 &,- 7 & \\
\hline & \multirow{3}{*}{6} & Recuento & 75 & 2 & 77 \\
\hline & & Frecuencia esperada & 74,8 & 2,2 & 77,0 \\
\hline & & Residuos corregidos & ,1 &,- 1 & \\
\hline & \multirow{3}{*}{7} & Recuento & 53 & 0 & 53 \\
\hline & & Frecuencia esperada & 51,5 & 1,5 & 53,0 \\
\hline & & Residuos corregidos & 1,3 & $-1,3$ & \\
\hline \multirow{2}{*}{ Total } & & Recuento & 1082 & 32 & 1114 \\
\hline & & Frecuencia esperada & 1082,0 & 32,0 & 1114,0 \\
\hline
\end{tabular}

Dado que el número de casillas con valores esperados inferiores a 5, sobrepasan el 20\% de las frecuencias esperadas $(28.6 \%$ de las casillas) de forma similar al caso anterior, el estadístico exacto de Fisher determinó un valor significativo de 0.012, que evidencia una asociación entre la bifurcación de los árboles en pie por rodal (Tabla 10). 
Tabla 10. Pruebas de Chi-cuadrado de la tabla de contingencia de la variable bifurcación de los

\begin{tabular}{|l|r|r|r|r|r|r|}
\hline & Valor & gl & $\begin{array}{c}\text { Sig. } \\
\text { asintótica } \\
\text { (bilateral) }\end{array}$ & $\begin{array}{c}\text { Sig. exacta } \\
\text { (bilateral) }\end{array}$ & $\begin{array}{c}\text { Sig. exacta } \\
\text { (unilateral) }\end{array}$ & $\begin{array}{c}\text { Probabilidad } \\
\text { en el punto }\end{array}$ \\
\hline Chi-cuadrado de Pearson & $18,902^{\mathrm{a}}$ & 6 &, 004 &, 008 & & \\
\hline Razón de verosimilitudes & 17,571 & 6 &, 007 &, 009 & & \\
\hline $\begin{array}{l}\text { Estadístico exacto de } \\
\text { Fisher }\end{array}$ & 14,690 & & &, $\mathbf{0 1 2}$ & & \\
\hline $\begin{array}{l}\text { Asociación lineal por } \\
\text { lineal }\end{array}$ &, $744^{\mathrm{b}}$ & 1 &, 388 &, 411 &, 207 &, 025 \\
\hline N de casos válidos & 1114 & & & & & \\
\hline
\end{tabular}

a. 4 casillas $(28,6 \%)$ tienen una frecuencia esperada inferior a 5 . La frecuencia mínima esperada es ,92.

b. El estadístico tipificado es, 863 .

\section{Análisis de contingencia de la relación ligera inclinación de los árboles en pie por rodal}

El análisis de los residuos corregidos para la variable ligera inclinación de los árboles en pie por rodal, indica dependencia provocada por una tendencia al incremento en los rodales 2 y 6 en sus valores de ausencia $(2,9$ y 3,1) y decremento en sus valores de presencia $(-2,9$ y $-3,1)$ pertenecientes al criterio evaluado respectivamente. En el caso del rodal 4 ocurre lo contrario, hay un decremento en su valor de ausencia $(-2,6)$ e incremento en su valor de presencia $(2,6)$ (Tabla 11). 
Tabla 11. Análisis de contingencia de la variable ligera inclinación de los árboles en pie por

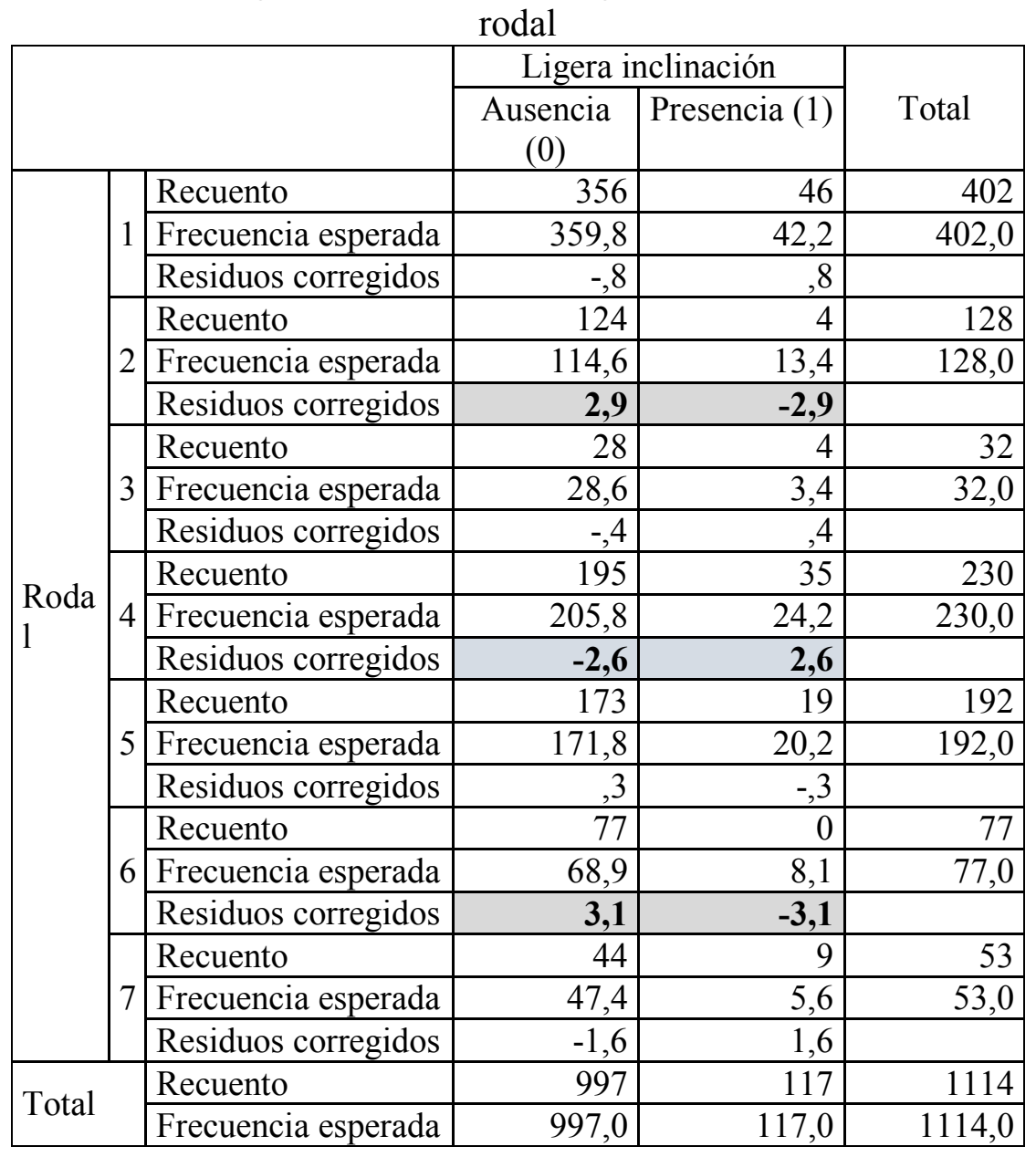

En lo referente al análisis de la relación entre la variable ligera inclinación de los árboles en pie por rodal, el p valor del Test Chi-cuadrado de Pearson indica una asociación significativa (pvalor $<\alpha=0.05$ ). El número de casillas con valores esperados inferiores a 5 , no sobrepasan el $10 \%$ de las frecuencias esperadas (7.1\% de las casillas), por tanto, se rechaza la hipótesis nula de independencia (Tabla 12). 
Tabla 12. Pruebas de Chi-cuadrado de la tabla de contingencia de la variable ligera inclinación de los árboles en pie por rodal

\begin{tabular}{|l|r|r|r|r|r|r|}
\hline & Valor & gl & $\begin{array}{c}\text { Sig. } \\
\text { asintótica } \\
\text { (bilateral) }\end{array}$ & $\begin{array}{c}\text { Sig. exacta } \\
\text { (bilateral) }\end{array}$ & $\begin{array}{l}\text { Sig. exacta } \\
\text { (unilateral) }\end{array}$ & $\begin{array}{c}\text { Probabilidad } \\
\text { en el punto }\end{array}$ \\
\hline Chi-cuadrado de Pearson & $24,843^{\mathrm{a}}$ & 6 &, 000 &.${ }^{\mathrm{b}}$ & & \\
\hline Razón de verosimilitudes & 34,496 & 6 &, 000 &.$^{\mathrm{b}}$ & & \\
\hline $\begin{array}{l}\text { Estadístico exacto de } \\
\text { Fisher }\end{array}$ & $\cdot^{\mathrm{b}}$ & & &. $\mathrm{b}$ & & \\
\hline $\begin{array}{l}\text { Asociación lineal por } \\
\text { lineal }\end{array}$ &, $000^{\mathrm{c}}$ & 1 &, 995 & 1,000 &, 509 &, 020 \\
\hline $\mathrm{N}$ de casos válidos & 1114 & & & & & \\
\hline
\end{tabular}

a. 1 casillas $(7,1 \%)$ tienen una frecuencia esperada inferior a 5. La frecuencia mínima esperada es 3,36.

b. No se puede efectuar el cálculo porque no hay suficiente memoria.

c. El estadístico tipificado es -,006.

\section{Análisis de contingencia de la relación inclinación de los árboles en pie por rodal}

El análisis de los residuos corregidos para la variable inclinación de los árboles en pie por rodal, refleja dependencia provocada por una tendencia al incremento en los rodales 2,4 y 7 en sus valores de ausencia $(4,2 ; 4,8$ y 2,5$)$ y decremento en los valores de presencia $(-4,2 ;-4,8$ y 2,5$)$ pertenecientes a la característica evaluada. En el caso del rodal 5 ocurre lo contrario, hay un decremento de su valor de ausencia $(-7,6)$ e incremento en su valor de presencia $(7,6)$ (Tabla 13). 
Tabla 13. Análisis de contingencia de la variable inclinación de los árboles en pie por rodal

\begin{tabular}{|c|c|c|c|c|c|}
\hline & & & Incli & nación & \\
\hline & & & $\begin{array}{c}\text { Ausencia } \\
(0)\end{array}$ & Presencia (1) & Total \\
\hline & & Recuento & 330 & 72 & 402 \\
\hline & 1 & $\begin{array}{l}\text { Frecuencia } \\
\text { esperada }\end{array}$ & 337,4 & 64,6 & 402,0 \\
\hline & & \begin{tabular}{|l|}
$\begin{array}{l}\text { Residuos } \\
\text { corregidos }\end{array}$ \\
\end{tabular} & $-1,3$ & 1,3 & \\
\hline & & Recuento & 124 & 4 & 128 \\
\hline & 2 & \begin{tabular}{|l|} 
Frecuencia \\
esperada
\end{tabular} & 107,4 & 20,6 & 128,0 \\
\hline & & \begin{tabular}{|l|} 
Residuos \\
corregidos
\end{tabular} & 4,2 & $-4,2$ & \\
\hline & & Recuento & 28 & 4 & 32 \\
\hline & 3 & \begin{tabular}{|l|}
$\begin{array}{l}\text { Frecuencia } \\
\text { esperada }\end{array}$ \\
\end{tabular} & 26,9 & 5,1 & 32,0 \\
\hline & & \begin{tabular}{|l}
$\begin{array}{l}\text { Residuos } \\
\text { corregidos }\end{array}$ \\
\end{tabular} & 6 &,- 6 & \\
\hline & & Recuento & 217 & 13 & 230 \\
\hline & 4 & \begin{tabular}{|l|} 
Frecuencia \\
esperada
\end{tabular} & 193,0 & 37,0 & 230,0 \\
\hline & & \begin{tabular}{|l|} 
Residuos \\
corregidos
\end{tabular} & 4,8 & $-4,8$ & \\
\hline & & Recuento & 126 & 66 & 192 \\
\hline & 5 & \begin{tabular}{|l|} 
Frecuencia \\
esperada
\end{tabular} & 161,1 & 30,9 & 192,0 \\
\hline & & \begin{tabular}{|l|} 
Residuos \\
corregidos \\
\end{tabular} & $-7,6$ & 7,6 & \\
\hline & & Recuento & 59 & 18 & 77 \\
\hline & 6 & \begin{tabular}{|l|} 
Frecuencia \\
esperada
\end{tabular} & 64,6 & 12,4 & 77,0 \\
\hline & & \begin{tabular}{|l}
$\begin{array}{l}\text { Residuos } \\
\text { corregidos }\end{array}$ \\
\end{tabular} & $-1,8$ & 1,8 & \\
\hline & & Recuento & 51 & 2 & 53 \\
\hline & 7 & \begin{tabular}{|l|}
$\begin{array}{l}\text { Frecuencia } \\
\text { esperada }\end{array}$ \\
\end{tabular} & 44,5 & 8,5 & 53,0 \\
\hline & & \begin{tabular}{|l|}
$\begin{array}{l}\text { Residuos } \\
\text { corregidos }\end{array}$ \\
\end{tabular} & 2,5 & $-2,5$ & \\
\hline & & Recuento & 935 & 179 & 1114 \\
\hline Total & & \begin{tabular}{|l|} 
Frecuencia \\
esperada
\end{tabular} & 935,0 & 179,0 & 1114,0 \\
\hline
\end{tabular}

En lo referente al análisis la variable inclinación de los árboles en pie por rodal, el Test Chicuadrado de Pearson indica una asociación significativa con un $p$-valor $<\alpha=0.05$ (Tabla 14). 
Tabla 14. Pruebas de Chi-cuadrado de la tabla de contingencia de la variable inclinación de los árboles en pie por rodal

\begin{tabular}{|l|r|r|r|r|r|r|}
\hline & Valor & gl & $\begin{array}{c}\text { Sig. } \\
\text { asintótica } \\
\text { (bilateral) }\end{array}$ & $\begin{array}{l}\text { Sig. exacta } \\
\text { (bilateral) }\end{array}$ & $\begin{array}{l}\text { Sig. exacta } \\
\text { (unilateral) }\end{array}$ & $\begin{array}{c}\text { Probabilidad } \\
\text { en el punto }\end{array}$ \\
\hline Chi-cuadrado de Pearson & $92,419^{\mathrm{a}}$ & 6 &, 000 &. $\mathrm{~b}$ & & \\
\hline Razón de verosimilitudes & 96,653 & 6 &, 000 &. $\mathrm{~b}$ & & \\
\hline $\begin{array}{l}\text { Estadístico exacto de } \\
\text { Fisher }\end{array}$ & $\cdot^{\mathrm{b}}$ & & &. $\mathrm{b}$ & & \\
\hline $\begin{array}{l}\text { Asociación lineal por } \\
\text { lineal }\end{array}$ & $2,633^{\mathrm{c}}$ & 1 &, 105 &, 108 &, 055 &, 004 \\
\hline $\mathrm{N}$ de casos válidos & 1114 & & & & & \\
\hline
\end{tabular}

a. 0 casillas $(0,0 \%)$ tienen una frecuencia esperada inferior a 5. La frecuencia mínima esperada es 5,14.

b. No se puede efectuar el cálculo porque no hay suficiente memoria.

c. El estadístico tipificado es 1,623 .

\section{Análisis de contingencia de la relación fuste recto de los árboles en pie por rodal}

El análisis de los residuos corregidos para la variable fuster recto de los árboles en pie, indica dependencia provocada por una tendencia al incremento en los rodales 5 , y 6 en sus valores de ausencia $(6,3$ y 2,2$)$ y decremento en sus valores de presencia $(-6,3$ y $-2,2)$ del criterio evaluado respectivamente. En el caso del rodal 2 sucede lo contrario, hay un decremento en su valor de ausencia $(-6,6)$ e incremento en su valor de presencia $(6,6)$ (Tabla 17). 
Tabla 17. Análisis de contingencia de la relación fuste recto de los árboles en pie por rodal

\begin{tabular}{|c|c|c|c|c|c|}
\hline & \multicolumn{2}{|c|}{ Fuste recto } & \multirow[b]{2}{*}{ Total } \\
\hline & & & $\begin{array}{c}\text { Ausencia } \\
(0)\end{array}$ & $\begin{array}{c}\text { Presencia } \\
\text { (1) }\end{array}$ & \\
\hline \multirow{21}{*}{ Rodal } & \multirow{3}{*}{1} & Recuento & 230 & 172 & 402 \\
\hline & & Frecuencia esperada & 229,1 & 172,9 & 402,0 \\
\hline & & Residuos corregidos & ,1 &,- 1 & \\
\hline & \multirow{3}{*}{2} & Recuento & 38 & 90 & 128 \\
\hline & & Frecuencia esperada & 73,0 & 55,0 & 128,0 \\
\hline & & Residuos corregidos & $-6,6$ & 6,6 & \\
\hline & \multirow{3}{*}{3} & Recuento & 16 & 16 & 32 \\
\hline & & Frecuencia esperada & 18,2 & 13,8 & 32,0 \\
\hline & & Residuos corregidos &,- 8 &, 8 & \\
\hline & \multirow{3}{*}{4} & Recuento & 122 & 108 & 230 \\
\hline & & Frecuencia esperada & 131,1 & 98,9 & 230,0 \\
\hline & & Residuos corregidos & $-1,4$ & 1,4 & \\
\hline & \multirow{3}{*}{5} & Recuento & 149 & 43 & 192 \\
\hline & & Frecuencia esperada & 109,4 & 82,6 & 192,0 \\
\hline & & Residuos corregidos & 6,3 & $-6,3$ & \\
\hline & \multirow{3}{*}{6} & Recuento & 53 & 24 & 77 \\
\hline & & Frecuencia esperada & 43,9 & 33,1 & 77,0 \\
\hline & & Residuos corregidos & 2,2 & $-2,2$ & \\
\hline & \multirow{3}{*}{7} & Recuento & 27 & 26 & 53 \\
\hline & & Frecuencia esperada & 30,2 & 22,8 & 53,0 \\
\hline & & Residuos corregidos &,- 9 & ,9 & \\
\hline \multirow{2}{*}{ Total } & & Recuento & 635 & 479 & 1114 \\
\hline & & Frecuencia esperada & 635,0 & 479,0 & 1114,0 \\
\hline
\end{tabular}

Al aplicar el Test Chi-cuadrado de Pearson para la tabla 17 de contingencia, se rechaza la hipótesis nula y se acepta que hay dependencia entre el variable fuste recto de los árboles en pie y los rodales al presentar un $\mathrm{p}$-valor $<\alpha=0.05$ como se muestra en la Tabla 18. 
Tabla 18. Pruebas de Chi-cuadrado de la tabla de contingencia de la variable fuste recto de los árboles en pie por rodal

\begin{tabular}{|c|c|c|c|c|c|c|}
\hline & Valor & $\mathrm{gl}$ & $\begin{array}{c}\text { Sig. } \\
\text { asintótica } \\
\text { (bilateral) }\end{array}$ & $\begin{array}{l}\text { Sig. exacta } \\
\text { (bilateral) }\end{array}$ & $\begin{array}{l}\text { Sig. exacta } \\
\text { (unilateral) }\end{array}$ & $\begin{array}{c}\text { Probabilidad } \\
\text { en el punto }\end{array}$ \\
\hline Chi-cuadrado de Pearson & $79,521^{2}$ & 6 &, 000 & $\mathrm{~b}$ & & \\
\hline Razón de verosimilitudes & 82,225 & 6 &, 000 & $\frac{f}{b}$ & & \\
\hline $\begin{array}{l}\text { Estadístico exacto de } \\
\text { Fisher }\end{array}$ & & & & b & & \\
\hline $\begin{array}{l}\text { Asociación lineal por } \\
\text { lineal }\end{array}$ & $13,264^{\circ}$ & 1 & 000 &, 000 &, 000 &, 000 \\
\hline $\mathrm{N}$ de casos válidos & 1114 & & & & & \\
\hline
\end{tabular}

a. 0 casillas $(0,0 \%)$ tienen una frecuencia esperada inferior a 5. La frecuencia mínima esperada es 13,76.

b. No se puede efectuar el cálculo porque no hay suficiente memoria.

c. El estadístico tipificado es $-3,642$.

\section{Agrupamiento de calidad de los árboles en pie por rodal}

Para el análisis de la calidad de los árboles por rodal se procedió a crear grupos de rodales que pudieran tratarse de manera homogénea en cuanto a un tratamiento futuro. Teniendo en cuenta que no todos los rodales presentan el mismo número de árboles, y que los métodos de clasificación basan su proceder en las distancias entre los valores de cada uno de los criterios, se procedió a trabajar con las frecuencias de presencia de los ocho atributos que conforman el estudio de la calidad (Tabla 19).

Tabla 19. Frecuencias de presencia de los ocho criterios evaluados para la variable calidad.

\begin{tabular}{|c|r|r|r|r|r|r|r|r|}
\hline Rodales & Ps & Ms & Tb & Bf & Li & I & Sin copa & Fr \\
\hline 1 & 0,256219 & 0 & 0,0199 & 0,027363 & 0,114428 & 0,179104 & 0,004975 & 0,427861 \\
\hline 2 & 0,210938 & 0,007813 & 0,007813 & 0,007813 & 0,03125 & 0,03125 & 0 & 0,703125 \\
\hline 3 & 0,1875 & 0 & 0,03125 & 0 & 0,125 & 0,125 & 0,03125 & 0,5 \\
\hline 4 & 0,130435 & 0,043478 & 0,069565 & 0,069565 & 0,152174 & 0,056522 & 0,004348 & 0,469565 \\
\hline 5 & 0,34375 & 0,072917 & 0,020833 & 0,192708 & 0,098958 & 0,34375 & 0 & 0,223958 \\
\hline 6 & 0,272727 & 0,064935 & 0,025974 & 0,181818 & 0 & 0,233766 & 0 & 0,311688 \\
\hline 7 & 0,339623 & 0 & 0 & 0 & 0,169811 & 0,037736 & 0 & 0,490566 \\
\hline
\end{tabular}

Al trabajar con valores de frecuencia relativa no fue necesario la estandarización de las variables, por lo que se procede a conformar los distintos conglomerados, usando para ello Conglomerados jerárquicos, Vinculación de Ward y Distancias Euclideas al cuadrado (Di Rienzo et al., 2009). Los resultados aparecen reflejados en el Dendrograma que se muestra en la figura 1, que indíca 
los rodales con similares características de calidad corresponden a: Rodales 1, 3 y 4 en un primer grupo, aunque el rodal 7 puede sumársele a este primer grupo al no existir mucha diferencia con los demás tres rodales. Sin embargo, si se considera el estudio de productividad reportada para la plantación de E. globulus, según lo reportado por Guallpa et al., (2016). En tal estudio se sitúa dentro del conglomerado de aprovechamiento conformado por los rodales 5 y 6 , más el rodal 7.

Situación que coincide en forma parcial con los resultados obtenidos en la evaluación de calidad de los árboles en pie en estudio, por tanto, debe integrarse al segundo grupo formado por los rodales 5 y 6, quedando como un rodal atípico en cuanto a los elementos de calidad de los árboles del rodal 2. En base a lo determinado, se asume que la calidad de los árboles, refleja por una parte la calidad de planta utilizada durante el establecimiento de la plantación y por otra, es el efecto de factores edafoclimáticos y de manejo silvicultural, que inciden en la formación de diferentes tipos de fustes (Salazar, 2008; Trujillo, 2012).

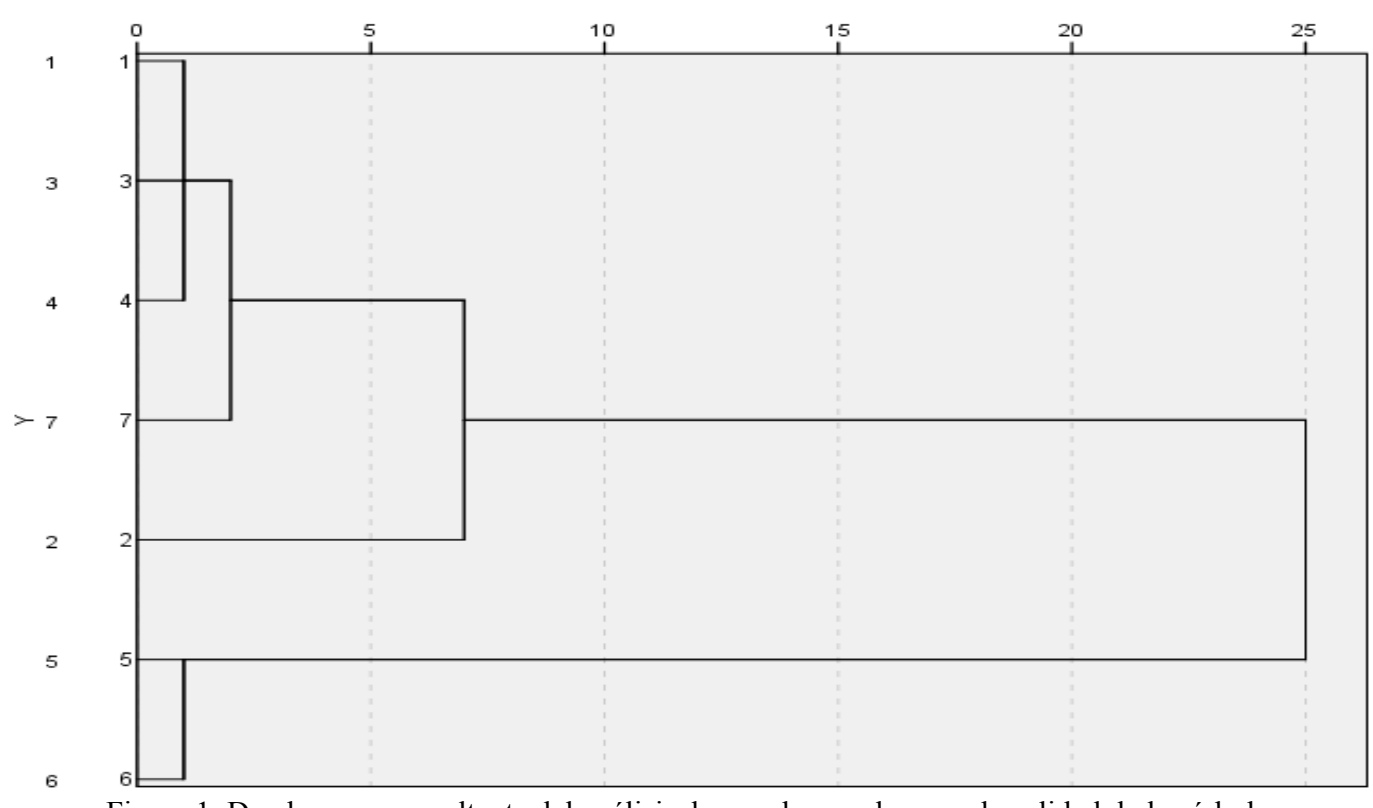

Figura 1. Dendrograma resultante del análisis de conglomerados para la calidad de los árboles (Método de Ward, distancia Euclidea al cuadrado) para los 7 rodales en estudio.

Los resultados obtenidos de esta investigación al evaluar de forma cualitativa a los árboles de $E$. globulus que forman parte de los siete rodales de la plantación ubicada en la Estación Experimental Tunshi, junto con la caracterización dasométrica reportada por Guallpa et al., (2016). En tal sentido, se dispone de información sobre la calidad, cantidad, y tipificación de los 
rodales (Rondeux, 2010), misma que sustenta y orienta, la estrategia de manejo técnico considerando el uso sostenible de la plantación en estudio para el área de aprovechamiento conformada por los rodales 5, 6 y 7 (FONAM, 2007).

Al contar con indicadores técnicos que facilitan la planificación técnica de las intervenciones silvícolas como parte del manejo de plantaciones que se desarrollan en las condiciones edafoclimáticas de la plantación en estudio. (Salazar, 2008). Mediante la ejecución de cortas de aprovechamiento de los árboles con base a un plan operativo y táctico para el manejo posterior de los rebrotes (Musálem, 2006). Por lo expuesto es importante aplicar el aprovechamiento y manejo de los rodales en mención, alternativa que coincide con lo sostenido por el INAB (2013), institución que sugiere el aprovechamiento de la madera en pie de un bosque plantado en la fase adulta. En el caso de los rodales 1,2,3 y,4 es conveniente aplicar tratamientos de carácter conservador a fin de garantizar su uso actual y permanente (Corporación de Estudios y Publicaciones, 2017).

\section{Conclusiones}

Los resultados obtenidos sobre la calidad de los árboles que forman parte del bosque plantado de E. globulus, indican que existe asociación significativa entre siete criterios utilizados para valorar a los árboles en pie por rodal. La creación de grupos de rodales en base a las frecuencias de presencia de los ocho atributos usados para la evaluación de los árboles en pie, determinó un primer grupo formado por los rodales $1,3 \mathrm{y} 4$; por su parte el rodal 7 presentó características similares al primer grupo, un segundo en cambio agrupa a los rodales 5 y 6 . El rodal 2 por sus características identificadas de calidad de los árboles dada su representación gráfica en el dendrograma elaborado, muestra una condición de rodal atípico.

Ejecutar el aprovechamiento de los árboles en los rodales 5, 6 y 7 en base a una planificación operativa, táctica y estratégica para el manejo silvicultural una vez que ocurra el crecimiento y desarrollo de nuevos rebrotes; y de carácter conservacionista para los rodales 1, 3, 4 teniendo en cuenta la agrupación de rodales, y los aspectos de carácter ambiental y económico al momento de tomar decisiones orientadas al manejo sostenible de la masa forestal, y de forma específica al rodal 2 . 


\section{Referencias Bibliográficas}

Alvarado, M., 2013. Guía de silvicultura: Análisis y prescripción de comportamientos en planes de manejo con fines de silvicultura y manejo forestal. Obtenido de http://icf.gob.hn/wpcontent/uploads/2015/08/GUIA-DE-SILVICULTURA.pdf

Corporación de Estudios y Publicaciones. 2017. Legislación Forestal. Quito, Ecuador: Tallere de la Corporación de Estudios y Publicaciones.

De la Fuente-Fernández, S., 2011. Tablas de Contingencia. Facultad de CienciasEconómicas y Empresariales. Universidad Autónoma de Madrid. Recuperado de:http://www.estadistica.net/ECONOMETRIA/CUALITATIVAS/CONTINGENCIA/tablascontingencia.pdf

De la Fuente-Fernández, S., 2011a. Análisis de Conglomerados. Facultad de CienciasEconómicas y Empresariales. Universidad Autónoma de Madrid. Recuperado de: http://www.fuenterrebollo.com/Economicas/ECONOMETRIA/SEGMENTACION/CONGLOMER ADOS/conglomerados.pdf

Di Rienzo, JA; Casanoves, F; Balzarini, MG; Conzalez, L; Tablada, M; Robledo, CW., 2009. InfoStat versión 2009. Grupo InfoStat, FCA, Universidad Nacional de Córdoba.

ETIFN. 2014. Manual de Campo. Procedimientos para la planificación, medición y registro de información del Inventario Forestal Nacional de Paraguay. FAO-PNUDPNUMAINFONA-SEAM-FAPI.

FAO. 2011. Diseños de muestreo de las Evaluaciones Forestales Nacionales. Recuperado de: http://www.fao.org/fileadmin/user_upload/national_forest_assessment/images/PDFs/Spanish/KR2_ ES_4_.pdf

Fondo Nacional del Ambiente. FONAM. 2007. Guía práctica para la instalación y manejo de plantaciones forestales. Lima, Perú.

García, G., 2016. Investigación comercial (4 ${ }^{\text {ta }}$ ed.). España: Esic.edu/editorial. 
González, A., 2017. Control de calidad de madera en pie y madera aserrada para el mercado de construcción del Grupo Empresarial El Almendro.Trabajo de pregrado.Universidad Nacional. Facultad de Ciencias de La Tierra y El Mar. Heredia, Costa Rica

Guallpa,M.,Rosero, S., Samaniego, M., \& Cevallos, E., 2016. Caracterización edáfica y dasométrica de una plantación de Eucalyptus globulus Labill y propuesta de manejo en la zona estepa espinosa Montano Bajo, Riobamba, Ecuador. EnfoqueUTE, 7 (3), 26-40.

Grupo Empresarial ENCE. 2009. La Gestión Forestal Sostenible y el Eucalipto. España: ENCE.

Hernández, J., 2014. Curso Regional de Regulación, Manejo y Salud Forestal. Chihuahua

Instituto Nacional de Bosques. INAB. 2013. Crecimiento y Productividad de Plantaciones Forestales de Teca en Guatemala. Guatemala.

Jiménez, C., 2008. Calidad y valoración de plantaciones forestales: aplicación práctica en cinco plantaciones de Vochysia guatemalensis Donn. Sm. (cebo) en las zonas Norte y Atlántica de Costa Rica. Kurú: Revista Forestal (Costa Rica) 5 (15):5 p.

López, P., \& Fachelli, S., 2015. Metodología de la Investigación Social Cuantitativa. Departament de Sociologia. Universitat Autónoma de Barcelona. Recuperado de https://ddd.uab.cat/pub/caplli/2015/131469/metinvsoccuan_cap3-6a2015.pdf

Ministerio de Agricultura, Ganadería, Acuacultura y Pesca. MAGAP. 2015. Programa de Incentivos para la Reforestación con Fines Comerciales. Guayaquil, Ecuador: MAGAP.

Murillo, O.,\& Badilla, Y., 2010. Calidad de la plantación forestal. ITCR, Cartago, C.R.67 p.

Musálem, M., 2006. Silvicultura de Plantaciones Forestales Comerciales. Chapingo, México: Universidad Autónoma Chapingo División de Ciencias Forestales.

Nájera, J. A., \& Hernández, H. E., 2008. Relaciones morfométricas de un bosque coetáneo de la región del Salto, Durango. Ra Ximhai (4 (1)), 69-81.

Nel, L., 2014. Estadística con SPSS 22. Lima: Macro.

Rojas, O., \& Murillo O., 2000. Calidad de las plantaciones de teca en la península de Nicoya, Costa Rica. Agronomía costarricense. 24(2): $65-75$ p. 
Rondeux, J., 2010. Medición de árboles y masas forestales. España: Mundi-Prensa.

Salazar, M., 2008. Propuesta de procedimientos para el establecimiento y seguimiento de parcelas permanentes de medición forestal en plantaciones beneficiarias del PINFOR. Unidad de Fomento Y Desarrollo Forestal, INAB. Guatemala.

Samaniego, C., 2013. Efecto de un incendio forestal en una plantación de Eucalyptus globulus Labill. subsp. globulus en Huaraz. Tesis de pregrado, Facultad de Ciencias Forestales, Universidad Nacional Agraria. Lima, Perú.

Sierra, R., 2012. Propuesta preliminar de un sistema de clasificación para el EcuadorContinental. Quito, Ecuador: Proyecto INEFAN/GEF-BIRF y EcoCiencia.

Trujillo, E., 2012 La Poda y su Influencia en la Calidad y Precio de la Madera. Revista el Mueble y la Madera, (75), 40 - 44. 